\title{
Coexistence of early onset sarcoidosis and partial interferon- $\gamma$ receptor 1 deficiency
}

\author{
Mustafa Çakan ${ }^{1}$, Gonca Keskindemirci² ${ }^{2}$ Çiğdem Aydoğmuş ${ }^{3}$, Hilal Ak1 ${ }^{4}$ Nevin Hatipoğlu ${ }^{5}$, \\ Aysel Kıyak ${ }^{6}$, Gönül Aydoğan7, Nuray Aktay-Ayaz ${ }^{1}$ \\ Clinics of ${ }^{1}$ Pediatric Rheumatology, ${ }^{2}$ Pediatrics, ${ }^{3}$ Pediatric Allergy and Immunology, ${ }^{6}$ Pediatric Nephrology, and ${ }^{7}$ Pediatric \\ Hematology and Oncology, Kanuni Sultan Süleyman Research and Training Hospital; ${ }^{4}$ Department of Pathology, Istanbul \\ University Cerrahpaşa Faculty of Medicine; and ${ }^{5}$ Clinic of Pediatric Infectious Diseases, Dr. Sadi Konuk Research and \\ Training Hospital, İstanbul, Turkey. E-mail: mustafacakan@hotmail.com
}

Received: 8th February 2016, Revised: 22nd April 2016, Accepted: 4th August 2016

\begin{abstract}
SUMMARY: Çakan M, Keskindemirci G, Aydoğmuş Ç, Akı H, Hatipoğlu N, Kıyak A, Aydoğan G, Aktay-Ayaz N. Coexistence of early onset sarcoidosis and partial interferon- $\gamma$ receptor 1 deficiency. Turk J Pediatr 2016; 58: 545-549.

Pediatric sarcoidosis comprises a spectrum of childhood granulomatous inflammatory conditions. Pathological hallmark of the disease is granuloma formation that is seen in the affected tissues and almost any organ or system can be involved. There are two forms of pediatric sarcoidosis. One is seen in older children and the clinical picture is very similar to that of adult sarcoidosis and the other one is seen in early childhood. Sarcoidosis in early childhood can be divided as Blau syndrome (familial form) and early onset sarcoidosis (sporadic form). In both of the diseases there is a defect in the NOD2/ CARD15 gene. The typical triad of early onset sarcoidosis is polyarthritis, dermatitis and uveitis. Interferon- $\gamma$ receptor 1 deficiency is caused by defects in the IFN $\gamma \mathrm{R} 1$ gene and non-tuberculosis mycobacterial pathogens are the leading causes of infections that start in early childhood. Herein we report a patient who presented with the symptoms of early onset sarcoidosis and also had partial interferon- $\gamma$ receptor 1 deficiency that presented with BCG-osis. In addition to anti-mycobacterial treatment, methotrexate and prednisolone were used in therapy.
\end{abstract}

Key words: BCG-osis, early onset sarcoidosis, interferon- $\gamma$ receptor 1 deficiency, macrophage activation syndrome, skeletal involvement.

Pediatric sarcoidosis is a rare multisystem granulomatous disorder. There are two distinct forms of sarcoidosis in children. Older children usually present with a multisystem disease similar to the adult sarcoidosis, with frequent hilar lymphadenopathy and pulmonary involvement. Blau syndrome (BS) and early onset sarcoidosis (EOS) constitute the familial (autosomal dominant) and sporadic forms of pediatric sarcoidosis that are usually seen under the age of four years and characterized by triad of polyarthritis, uveitis and rash ${ }^{1,2}$. The typical triad of rash, arthritis and uveitis is seen in around $75 \%$ of the patients ${ }^{2}$. The responsible gene in both of these disorders is the NOD2/ CARD15 gene and gain of function mutations in this gene cause increased NF- $\kappa B$ activity leading to oversecretion of proinflammatory cytokines and systemic inflammation ${ }^{3}$. The initial symptoms of the EOS are seen around two years of age and reddish to brown, scaly rash is being the first symptom. The clinical picture of the EOS is not restricted to the skin, joint and eye. Fever, nephritis, interstitial pneumonitis, peripheral lymphadenopathy, pericarditis, cranial neuropathies, hepatic granulomatous infiltration are other manifestations of the disease $\mathrm{e}^{1,2,4,5}$.

Mendelian susceptibility to mycobacterial diseases is caused by genetic defects in the IL-12/IFN- $\gamma$ pathway ${ }^{6}$. Patients with interferongamma (IFN-y) receptor deficiency have increased susceptibility to systemic infections with intracellular microorganisms, especially weakly virulent nontuberculous mycobacteria including Bacillus Calmette-Guerin (BCG) vaccine strain. In countries where BCG vaccine 


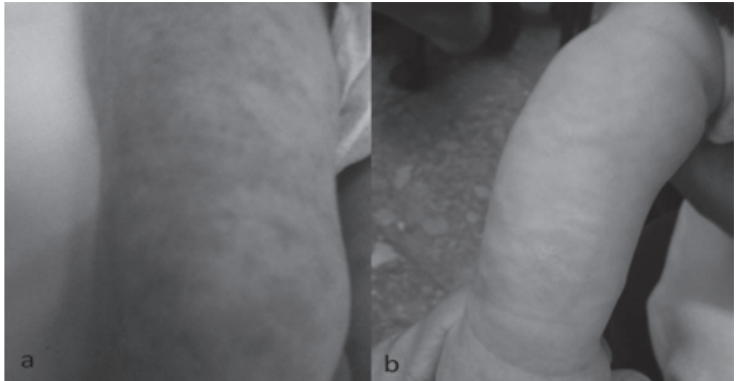

Fig. 1. The rash on the leg (a) and on the arm (b)

is employed routinely, patients with IFN- $\gamma$ receptor deficiency may develop disseminated BCG-osis during infancy or early childhood. Clinical features of BCG-osis are nonspecific and may overlap with the signs and symptoms of EOS such as fever, diarrhea, weight loss, generalized lymphadenopathy, diffuse cutaneous lesions, and hepatosplenomegaly. ${ }^{7,8}$ This report describes a patient with EOS. He was found to have partial IFN- $\gamma$ receptor 1 deficiency that presented with BCG-osis. In addition to antimycobacterial treatment, immunosuppressive treatment was used in the therapy.

\section{Case Report}

A 17-month-old male patient, born to firstcousin parents was admitted to our hospital with the complaints of fever, rash, and abdominal distention lasting for 6 months. The patient did not have any complaints until 11 months of age. After then, he was hospitalized for 6 months in two different centers because of the quotidian fever and rash. He was the first child of the family, and family history was unremarkable. On physical examination he had scaly, reddish to brown, maculopapular rash on the face, trunk and extremities (Fig. 1). Liver and spleen were 2 $\mathrm{cm}$ palpable below the costal margin. There were bilateral cervical and axillary lymph nodes under $1 \mathrm{~cm}$ in diameter. The patient did not have arthritis or uveitis. Complete blood count and acute phase reactants showed: leukocytes $11,500 / \mathrm{mm}^{3}$, hemoglobin $8.7 \mathrm{~g} / \mathrm{dl}$, platelets $197,000 / \mathrm{mm}^{3}$, erythrocyte sedimentation rate (ESR) $90 \mathrm{~mm} /$ hour, C-reactive protein (CRP) $122 \mathrm{mg} / \mathrm{L}$ (normal: < $5 \mathrm{mg} / \mathrm{L}$ ). He had mild hypertransaminasemia (AST 77 $\mathrm{U} / \mathrm{L}$, ALT $50 \mathrm{U} / \mathrm{L}$ ), and albumin was $2.8 \mathrm{~g} /$ dl. On X-ray and computerized tomography (CT) of the thorax nonspecific consolidations on lung parenchyma and multiple expansile, lytic lesions on the left 5th, right 6th and 8th costae and left humerus were observed (Fig. 2). On ultrasound and CT examinations of the abdomen, multiple hypodense lesions in the liver and bilateral multiple kidney stones were detected. Immunodeficiency, tuberculosis, malignancy and EOS were in the differential diagnosis. Purified protein derivative tuberculin skin test was $17 \mathrm{~mm}$, and quantiferon assay was borderline positive. Anti-tuberculosis treatment consisting of isoniazid $(15 \mathrm{mg} / \mathrm{kg} /$ day, po), rifampicin $(20 \mathrm{mg} / \mathrm{kg} /$ day, po) and pyrazinamide $(25 \mathrm{mg} / \mathrm{kg} /$ day, po) was initiated. Acido-resistant bacilli studies were negative on fasting gastric fluid and cerebrospinal fluid examinations. Mycobacterium bovis (BCG-vaccine

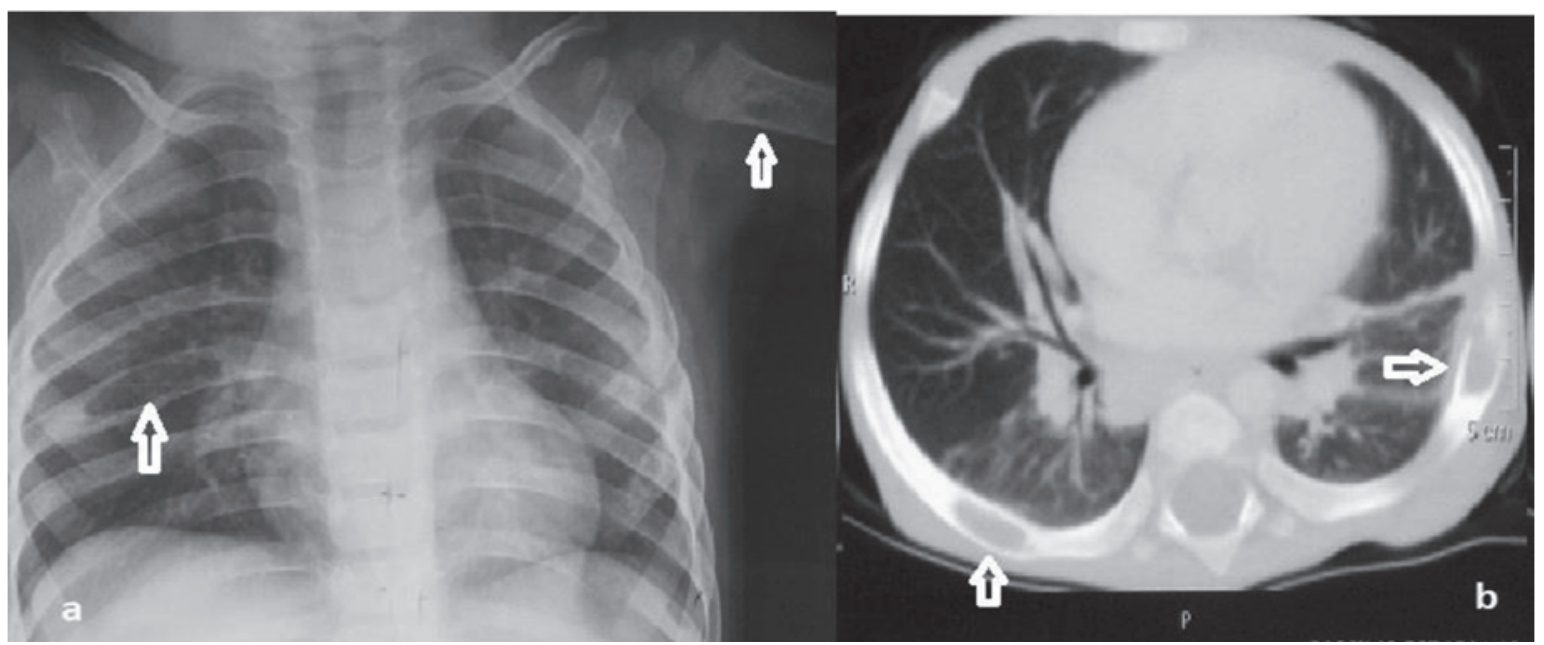

Fig. 2. Multiple expansile lytic lesions on the X-ray (a) and CT (b) of the thorax 


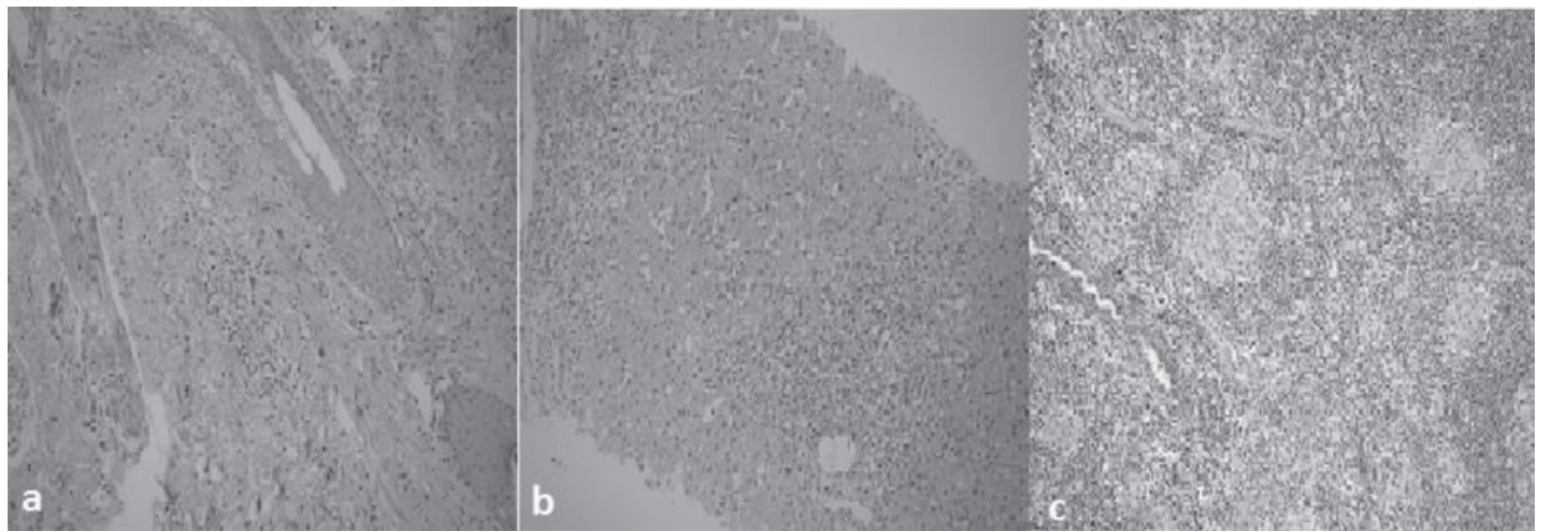

Fig. 3. Granuloma formations on the skin (a), liver (b) and lymph node (c) specimens

type) was isolated from gastric fluid. The patient was diagnosed as BCG-osis. Streptomycin (20 $\mathrm{mg} / \mathrm{kg} /$ day, im) and ethambutol $(20 \mathrm{mg} / \mathrm{kg} /$ day, po) were added and pyrazinamide was discontinued. Serum immunoglobulin levels, lymphocyte subtype and burst-suppression studies were normal. Biopsies that were taken from the liver, skin and cervical lymph node and bone marrow were reevaluated. Mononuclear cell infiltration and granuloma formation were observed in all biopsies (Fig. 3). Ziehl-Nielsen, S-100 and Langerin stains were negative.

On the 2nd week of hospitalization, the patient developed persistent fever, dyspnea, pancytopenia (leukocytes 2,000/mm3, platelets $6,000 / \mathrm{mm}^{3}$, hemoglobin $5.6 \mathrm{~g} /$ dl), hypoalbuminemia (albumin $2.1 \mathrm{~g} / \mathrm{dl}$ ), hyperferritinemia (ferritin $45.000 \mathrm{ng} / \mathrm{ml}$ ), hypofibrinogenemia (fibrinogen $40 \mathrm{mg} / \mathrm{dl}$ ), high CRP $(155 \mathrm{mg} / \mathrm{L})$ and low ESR $(20 \mathrm{~mm} / \mathrm{hr})$. The patient was transferred to pediatric intensive care unit with the provisional diagnosis of macrophage activation syndrome (MAS). On bone marrow examination hemophagocytosis was not observed. In addition to the supportive care, high dose intravenous methylprednisolone treatment $(30 \mathrm{mg} / \mathrm{kg} /$ day $)$ was given for three days. The clinical and laboratory parameters of the patient improved in two weeks. On the 6th week, the patient developed central type left facial nerve palsy. The symptoms resolved spontaneously in one week. Serum angiotensin-converting enzyme level was found to be $61.6 \mathrm{U} / \mathrm{L}$ (normal: $8-52 \mathrm{U} / \mathrm{L}$ ). On the work-up for renal stones, serum parathyroid hormone, vitamin $\mathrm{D}$, calcium, alkaline phosphatase and magnesium levels were normal. No hyperoxaluria or hypocitraturia but hypercalciuria were detected in 24-hour urine studies. Hydrochlorothiazide treatment (2 $\mathrm{mg} / \mathrm{kg} /$ day, po) was initiated.

As no clinical and laboratory improvement were achieved (persistence of fever, rash, and high acute phase reactants) by antimycobacterial treatment and by the presence of granuloma formation on the biopsy specimens, hypercalciuria, renal calculi, and multiple bone lesions, methotrexate $(10 \mathrm{mg} /$ $\mathrm{m}^{2} /$ week, po) and prednisolone (1 $\mathrm{mg} / \mathrm{kg}$ / day, po) were started on the 4 th month of hospitalization with the diagnosis of EOS. By this treatment, fever and rash resolved on the 2 nd week and laboratory parameters were normalized on the 4th week. After six months of hospitalization he was discharged with anti-mycobacterial drugs, hydrochlorothiazide, methotrexate and prednisolone. On the 6th month of methotrexate treatment, the doses of methotrexate and prednisolone were lowered to minimum levels. One month later, he developed new bone lesions on costae and iliac crest. The child did not have fever or rash but CRP was $88 \mathrm{mg} / \mathrm{L}$ and ESR was $68 \mathrm{~mm} /$ hour. The doses of methotrexate and prednisolone were increased and the lesions resolved in 2 months. Anti-mycobacterial medications were given for a year. The child had chickenpox when he was 3-years-old and lesions healed in one week without any complication. On genetic analysis, heterozygous missense mutation (P268S) in NOD2 gene, and homozygous mutation (I37T) in IFNGR1 gene that caused partial IFNyR1 deficiency were found. The patient has been followed for 4.5 years and currently taking no medication. 


\section{Discussion}

Blau syndrome and EOS are classified under the granulomatous autoinflammatory diseases. ${ }^{9}$ $\mathrm{BS}$ is the familial and EOS is the sporadic form of the same disease with similar genetic and clinical background. The typical triad of EOS is rash, arthritis and uveitis and the pathological hallmark is granuloma formation in affected tissues. ${ }^{4,5}$ In the report of Rose et al. ${ }^{5} 34$ of 45 patients $(75.5 \%)$ with BS/EOS had typical triad. In the study of Arostegui et al. ${ }^{4} 5$ of 12 patients $(41.7 \%)$ had typical triad. In addition to the classical triad, patients with EOS may have fever, nephritis, interstitial pneumonitis, peripheral lymphadenopathy, pericarditis, transient cranial neuropathies (especially facial nerve), hepatic granulomatous infiltration, and renal calcification. 1,2,4,5,10 Our patient's initial symptom was rash and fever that started when he was 11-months-old. Okafuji et al. ${ }^{2}$ found that 11 of 20 patients with BS/EOS had fever and 5 had persistent fever. Besides fever, our patient had findings described in the literature, like hepatosplenomegaly, cervical and axillary lymphadenopathy, renal calculi, interstitial pneumonitis, transient facial nerve palsy and granulomatous liver involvement confirmed by biopsy. As far as we know bone lesions and MAS recorded in this patient were not described before. We also think that bone lesions may be secondary to accompanying mycobacterial infection and cause of MAS may be an occult intervening infection. Although arthralgia was present, our case never developed classical boggy tenosynovitis of EOS. In the report of Rose et al..$^{5} 2$ out of 45 patients did not develop any arthritis. Uveitis that can lead to blindness is the most serious problem in EOS patients. Fink et al. ${ }^{11}$ followed 6 patients with EOS with a median duration of 14 years and 4 developed blindness. Uveitis is the other component of the triad that did not develop in this patient. In the study of Okafuji et al. ${ }^{2}$ median age at onset of uveitis was found to be 4.5 years and also they described 20 BS/ EOS patients, 2 without uveitis. Although uveitis of EOS may develop under treatment, development of uveitis in this patient might have been prevented by immunosuppressive treatment. Heterozygous missense mutation (P268S) in NOD2 gene that was found in our patient was described as a pathogenic mutation in EOS/BS patients in the literature ${ }^{12}$.
IFN- $\gamma$ is produced by $\mathrm{T}$ cells and natural killer cells and induces macrophage activation, resulting in increased production of interleukin- 1 and TNF- $\alpha$, enhanced antigen presentation and increased production of nitric oxide and reactive-oxygen intermediates. IFN- $\gamma$ receptor deficiency causes increased susceptibility to systemic infections with intracellular organisms, including mycobacteria, salmonella, and leishmania. Patients with this defect are vulnerable to weakly virulent nontuberculous mycobacteria including Bacillus Calmette-Guerin (BCG) vaccine strain. Patients with complete deficiency develop severe, recurring disseminated mycobacterial disease in infancy or early childhood that can cause to mortality. Patients with partial deficiency tend to have a less severe phenotype and present with localized or disseminated BCG or nontuberculous mycobacteria infections. Most patients develop multifocal osteomyelitis. ${ }^{6,7}$ Mycobacterial infections as triggering factor of granuloma formation in sarcoidosis is suspected for many years. ${ }^{13,14}$ This patient had proven mycobacterial infection. Mycobacterium bovis (BCG-vaccine type) was isolated from gastric fluid. Genetic analysis showed that he had partial IFN- $\gamma$ receptor deficiency, and functional studies showed impaired but not abolished response to IFN- $\gamma$. Homozygous mutation (I37T) in IFNGR1 gene that was found in our patient was not described previously in the literature, so this was a novel mutation. ${ }^{15}$

There are few case reports in which authors speculated that mycobacterial infections could trigger the symptoms of EOS. Osborne et al. ${ }^{16}$ presented a patient with juvenile sarcoidosis whose rash started around BCG vaccination site, and they thought that $M$. bovis might be responsible from the development of sarcoidosis. The patient presented by Sakai et al. ${ }^{17}$ had uveitis when he was 5 years old, developed polyarthritis when he was 15 , and at 30 years of age skin rash had developed on the extremities after BCG vaccination

Our case has a few distinctive features of EOS in addition to several manifestations described in the literature, but has also multiple skeletal involvement and MAS. Although the cause of MAS may be an intervening infection and not EOS, recurrence of bone lesions after tapering of the immunosuppressive therapy made us 
think that multiple bone lesions might be due to EOS. To the best of our knowledge this is the first case of EOS with partial IFN- $\gamma$ receptor deficiency described in the literature. In conclusion, in children with multiorgan involvement EOS should be kept in mind, and we think that mycobacterial infections may trigger the symptoms of EOS.

\section{Acknowledgement}

NOD2/CARD15 gene analysis was done at Ege University, Department of Pediatrics, Molecular Medicine Laboratory, İzmir, Turkey by Afig Berdeli and colleagues and IFNGR1 gene analysis was done at Laboratoire de Génétique Humaine des Maladies Infectieuses, Paris, France by Jacinta Cecilia Bustamante and colleagues. We would like to thank to both centers for their collaboration.

\section{REFERENCES}

1. Rosé CD, Wouters CH. Pediatric sarcoidosis. In: Cassidy JT, Petty RE, Laxer ML, Lindsey CB (eds). Textbook of Pediatric Rheumatology (6th ed). Philadelphia: Elsevier, 2011: 544-551.

2. Okafuji I, Nishikomori R, Kanazawa N, et al. Role of the NOD2 genotype in the clinical phenotype of Blau syndrome and early-onset sarcoidosis. Arthritis Rheum 2009; 60: 242-250.

3. Miceli-Richard C, Lesage S, Rybojad M, et al. CARD15 mutations in Blau syndrome. Nat Genet 2001; 29: 19-20.

4. Aróstegui JI, Arnal C, Merino R, et al. NOD2 geneassociated pediatric granulomatous arthritis: clinical diversity, novel and recurrent mutations, and evidence of clinical improvement with interleukin-1 blockade in a Spanish cohort. Arthritis Rheum 2007; 56: 38053813.

5. Rosé CD, Aróstegui JI, Martin TM, et al. NOD2associated pediatric granulomatous arthritis, an expanding phenotype: study of an international registry and a national cohort. Arthritis Rheum 2009; 60: 1797-1803.
6. Al-Muhsen S, Casanova JL. The genetic heterogeneity of mendelian susceptibility to mycobacterial diseases. J Allergy Clin Immunol 2008; 122: 1043-1051.

7. Dorman SE, Picard C, Lammas D, et al. Clinical features of dominant and recessive interferon gamma receptor 1 deficiencies. Lancet 2004; 364: 2113-2121.

8. Edeer Karaca N, Boisson-Dupuis S, Aksu G, et al. Granulomatous skin lesions, severe scrotal and lower limb edema due to mycobacterial infections in a child with complete IFN- $\gamma$ receptor-1 deficiency. Immunotherapy 2012; 4: 1121-1127.

9. Caso F, Rigante D, Vitale A, et al. Monogenic autoinflammatory syndromes: state of the art on genetic, clinical, and therapeutic issues. Int J Rheumatol 2013; 2013: 513782.

10. Kanazawa N, Okafuji I, Kambe N, et al. Early-onset sarcoidosis and CARD15 mutations with constitutive nuclear factor- $\mathrm{\kappa B}$ activation: common genetic etiology with Blau syndrome. Blood 2005; 105: 1195-1197.

11. Fink CW, Cimaz R. Early onset sarcoidosis: not a benign disease. J Rheumatol 1997; 24: 174-177.

12. Caso F, Galozzi P, Costa L, Sfriso P, Cantarini L, Punzi L. Autoinflammatory granulomatous diseases: from Blau syndrome and early-onset sarcoidosis to NOD2-mediated disease and Crohn's disease. RMD Open 2015; 1: e000097.

13. Saboor SA, Johnson NM, McFadden J. Detection of mycobacterial DNA in sarcoidosis and tuberculosis with polymerase chain reaction. Lancet 1992; 339: 1012-1015.

14. Mangiapan G, Hance AJ. Mycobacteria and sarcoidosis: an overview and summary of recent molecular biological data. Sarcoidosis 1995; 12: 20-37.

15. Bustamante J, Boisson-Dupuis S, Abel L, Casanova JL. Mendelian susceptibility to mycobacterial disease: genetic, immunological, and clinical features of inborn errors of IFN- $\gamma$ immunity. Semin Immunol 2014; 26 : 454-470.

16. Osborne GE, Mallon E, Mayou SC. Juvenile sarcoidosis after BCG vaccination. J Am Acad Dermatol 2003; 48: 99-102.

17. Sakai H, Ito S, Nishikomori R, et al. A case of earlyonset sarcoidosis with a six-base deletion in the NOD2 gene. Rheumatology 2010; 49: 194-196. 